\title{
Systemic Radiopharmaceutical Therapy of Pheochromocytoma and Paraganglioma
}

\author{
Jorge A. Carrasquillo ${ }^{1,2}$, Clara C. Chen ${ }^{3}$, Abhishek Jha ${ }^{4}$, Karel Pacak ${ }^{4}$, Daniel A. Pryma ${ }^{* 5}$, and Frank I. Lin ${ }^{* 2}$ \\ ${ }^{1}$ Molecular Imaging and Therapy Service, Memorial Sloan Kettering Cancer Center, New York, New York; ${ }^{2}$ Molecular Imaging \\ Branch, National Cancer Institute, Bethesda, Maryland; ${ }^{3}$ Department of Radiology, Clinical Center, National Institutes of Health, \\ Bethesda, Maryland; ${ }^{4}$ Section on Medical Neuroendocrinology, National Institute of Child Health and Human Development, National \\ Institutes of Health, Bethesda, Maryland; and ${ }^{5}$ Department of Radiology, Hospital of University of Pennsylvania, Philadelphia, \\ Pennsylvania
}

\begin{abstract}
Learning Objectives: On successful completion of this activity, participants should be able to (1) describe the clinical features of pheochromocytoma and paraganglioma, including common symptoms and signs, management options, imaging findings, and the role of genetic and hormonal biomarkers; (2) identify and compare radiopharmaceutical agents available for treatment of metastatic or inoperable pheochromocytoma and paraganglioma; and (3) identify differences between low-specific-activity and high-specific-activity ${ }^{131}$ I-MIBG.

Financial Disclosure: This research was funded in part through the NIH/National Cancer Institute Cancer Center support grant P30 CA008748 and Eunice Kennedy Shriver National Institutes of Child Health and Human Development grant Z1AHD008735, NIH. Daniel Pryma receives research funding from Siemens and 511 Pharma and consulting fees from Siemens, 511 Pharma, and Bayer. The authors of this article have indicated no other relevant relationships that could be perceived as a real or apparent conflict of interest.

CME Credit: SNMMI is accredited by the Accreditation Council for Continuing Medical Education (ACCME) to sponsor continuing education for physicians. SNMMI designates each JNM continuing education article for a maximum of 2.0 AMA PRA Category 1 Credits. Physicians should claim only credit commensurate with the extent of their participation in the activity. For CE credit, SAM, and other credit types, participants can access this activity through the SNMMI website (http://www.snmmilearningcenter.org) through September 2024.
\end{abstract}

Whereas benign pheochromocytomas and paragangliomas are often successfully cured by surgical resection, treatment of metastatic disease can be challenging in terms of both disease control and symptom control. Fortunately, several options are available, including chemotherapy, radiation therapy, and surgical debulking. Radiolabeled metaiodobenzylguanidine (MIBG) and somatostatin receptor imaging have laid the groundwork for use of these radiopharmaceuticals as theranostic agents. ${ }^{131}$ I-MIBG therapy of neuroendocrine tumors has a long history, and the recent approval of high-specific-activity ${ }^{131}$ I-MIBG for metastatic or inoperable pheochromocytoma or paraganglioma by the U.S. Food and Drug Administration has resulted in general availability of, and renewed interest in, this treatment. Although reports of peptide receptor radionuclide therapy of pheochromocytoma and paraganglioma with ${ }^{90} \mathrm{Y}$ - or ${ }^{177}$ Lu-DOTA conjugated somatostatin analogs have appeared in the literature, the approval of ${ }^{177}$ Lu-DOTATATE in the United States and Europe, together with National Comprehensive Cancer Network guidelines suggesting its use in patients with metastatic or inoperable pheochromocytoma and paraganglioma, has resulted in renewed interest. These agents have shown evidence of efficacy as palliative treatments in patients with metastatic or inoperable pheochromocytoma or paraganglioma. In this continuing medical education article, we discuss the therapy of pheochromocytoma and paraganglioma with ${ }^{131} \mathrm{I}-\mathrm{MIBG}$ and ${ }^{90} \mathrm{Y}$ - or ${ }^{177}$ Lu-DOTAsomatostatin analogs.

Key Words: pheochromocytoma; paraganglioma; somatostatin; MIBG; DOTATATE; peptide receptor radionuclide therapy

Received Nov. 2, 2020; revision accepted Feb. 16, 2021.

For correspondence or reprints, contact Jorge A. Carrasquillo (carrasj1@ mskcc.org).

${ }^{*}$ Contributed equally to this work.

COPYRIGHT (C) 2021 by the Society of Nuclear Medicine and Molecular Imaging.
J Nucl Med 2021; 62:1192-1199

DOI: 10.2967/jnumed.120.259697

$\mathbf{P}$

heochromocytomas and paragangliomas are rare neuroendocrine tumors (NETs) typically arising in chromaffin tissue, with an overall incidence of 0.4-2.1 cases per million people (1), although higher incidences may be seen in pathology series. In accordance with World Health Organization guidelines, these tumors are classified as paragangliomas (2); we will refer to them as pheochromocytomas when they arise from the adrenal medulla and as paragangliomas when extraadrenal. Pheochromocytoma and paraganglioma most frequently arise from sympathetic tissues. Those arising from parasympathetic tissue are most often in the head and neck. In our previous article, we reviewed the genetics, phenotype, presentation, and imaging characteristics of pheochromocytoma and paraganglioma (3).

Patients with pheochromocytoma and paraganglioma often present with symptoms of elevated catecholamines, depending on the genotype. Tumors of sympathetic origin often secrete elevated levels of epinephrine or norepinephrine or of their metabolites metanephrine and normetanephrine. Tumors of parasympathetic origin may secrete elevated levels of dopamine or its metabolite 3-methoxy-tyramine but are often asymptomatic. A small percentage of pheochromocytoma and paraganglioma are biochemically silent.

Although only $2 \%-26 \%$ of pheochromocytomas and paragangliomas are metastatic (4), benign and malignant tumors cannot be differentiated histologically; thus, the term metastatic rather than malignant is used when tumor is found outside the normal sites of chromaffin or paraganglia tissues (2). Common sites of metastatic disease include the lymph nodes, bone, liver, and lung (4). Currently, 
systemic radiopharmaceutical therapies (RPT) for pheochromocytoma and paraganglioma include ${ }^{131}$ I-metaiodobenzylguanidine (iobenguane, ${ }^{131}$ I-MIBG) of low specific activity (LSA) or high specific activity (HSA) and peptide receptor radionuclide therapy (PRRT) with ${ }^{90} \mathrm{Y}$ - or ${ }^{177} \mathrm{Lu}$-DOTA-somatostatin analogs.

\section{GENERAL CONSIDERATIONS FOR SYSTEMIC RPT IN PHEOCHROMOCYTOMA AND PARAGANGLIOMA}

Although most patients with benign pheochromocytoma and paraganglioma are cured with surgical resection, and local control of head and neck pheochromocytoma and paraganglioma is usually achieved with definitive external-beam radiotherapy, approximately one third are not cured and require long-term follow-up. Patients with benign disease have a survival rate similar to that of healthy individuals, whereas patients with metastatic disease have a 5-y survival rate of $55 \%-92 \%(4,5)$. The wide differences in survival of pheochromocytoma and paraganglioma patients are related to several variables, including genetic status, size of primary tumor, biochemical phenotype, and presence of metastatic disease at initial diagnosis $(6,7)$. Long-term stability over $15 \mathrm{y}$ is not uncommon. Since current treatments of metastatic disease are rarely curative, a watch-and-wait approach is often appropriate, reserving therapy for progressive or symptomatic disease that is not responsive to medical treatment or amenable to surgical resection (8). Systemic treatment is also an option for inoperable nonmetastatic disease that is progressing or has refractory symptoms.

The goals of treatment are palliative, including prevention of progression and improvement of symptoms related to tumor burden or catecholamine release. For systemic treatment, the most common chemotherapy regimen is cisplatin, vinblastine, and dacarbazine, which may be preferred for rapidly progressing disease (9). Tyrosine kinase inhibitors that target the vascular endothelial growth factor pathway, such as sunitinib, may have efficacy (10). External radiation for localized symptomatic disease is generally successful for local control (11). For less urgent treatment, RPT with LSA or HSA ${ }^{131}$ I-MIBG and PRRT are available. Except for HSA ${ }^{131} \mathrm{I}-\mathrm{MIBG}$, none of these approaches are currently approved by the U.S. Food and Drug Administration (FDA) for pheochromocytoma and paraganglioma.

\section{PATIENT PREPARATION BEFORE RPT}

When catecholamines or their metabolites (metanephrines) are elevated, experts recommend catecholamine blockade before invasive intervention or RPT; the target is blood pressure control, but often labile hypertension persists (12). Typically, first-line treatment consists of $\alpha$-blockade (e.g., phenoxybenzamine or doxazocin). A concomitant $\beta$-blocker (e.g., atenolol or metoprolol) is often required but should never be used without adequate $\alpha$-blockade, because unopposed $\alpha$-adrenergic receptor stimulation could precipitate a hypertensive crisis. Occasionally, addition of metyrosine is needed to block catecholamine synthesis.

Catecholamine release symptoms (hypertension, tachycardia, palpitation, headache, chest pain, tachypnea, sweating, cutaneous flushing, anxiety, and the more severe or life-threatening symptoms of stroke and myocardial infarction) $(13,14)$ are possible during PRRT and MIBG infusion or in the early posttreatment period $(13,15)$. Gonias et al. (15) reported acute hypertension in approximately $14 \%$ of subjects and in $15 \%$ of high-radioactivity LSA ${ }^{131}$ I-MIBG treatments, occurring approximately $30 \mathrm{~min}$ after initiation of infusion and requiring treatment. Sometimes hypertension recurred on repeat treatment. Although it is difficult to predict who will develop a hypertensive response to RPT, patients who are biochemically silent will not. Although no acute hypertensive crises were observed with HSA ${ }^{131}$ I-MIBG, the package insert indicates an $11 \%$ incidence of grade 3 or 4 hypertension within $24 \mathrm{~h}$ of infusion. Physicians administering RPT should be prepared to treat blood pressure elevation with oral or parenteral antihypertensives (e.g., phentolamine). Close coordination with other medical specialties, including intensive care, is prudent.

Because of the teratogenic effects of ionizing radiation, patients receiving RPT should avoid conception ( 7 mo for women and 4 mo for men) and must not be pregnant or breastfeeding during treatment. Patients must also be willing and able to follow radiation safety precautions.

\section{POST-RPT RADIATION PROTECTION}

In the United States, the Nuclear Regulatory Commission regulations allow for discharge of patients receiving RPT if exposure to others is not likely to exceed $0.5 \mathrm{cGy}$, and written instructions must be provided to outpatients if the dose is likely to be more than 0.1 cGy. Other countries require RPT to be administered as an inpatient procedure or use different activity levels as the cutoff. Even if release criteria for outpatient administration are met, inpatient treatment may be prudent for some patients. In the United States, outpatient therapies are often performed when LSA ${ }^{131} \mathrm{I}$ MIBG therapy is less than $9.25 \mathrm{GBq}$ or no more than $7.4 \mathrm{GBq}$ of ${ }^{177}$ Lu-DOTATATE, whereas with the recommended dose of approximately $18.5 \mathrm{GBq}$ of HSA ${ }^{131} \mathrm{I}-\mathrm{MIBG}$, treatment must be inpatient.

\section{${ }^{131}$ I-MIBG THERAPY}

${ }^{131}$ I-MIBG was first described by Wieland et al. for imaging the adrenal medulla (16). At present, ${ }^{123} \mathrm{I}-\mathrm{MIBG}$ is preferred for imaging and ${ }^{131}$ I-MIBG for therapy. Currently, ${ }^{131}$ I-MIBG is seldom used for imaging pheochromocytoma and paraganglioma except for performing dosimetry and for documenting posttreatment targeting. The physical properties of ${ }^{131}$ I-MIBG make it a useful therapeutic agent, including a short-range 606-keV $\beta$-emission and a $364-\mathrm{keV} \gamma$-ray that allows imaging and dosimetry. Furthermore, its 8.02-d half-life allows flexibility for labeling and shipping.

MIBG is a derivative of guanethidine and a substrate for the norepinephrine (noradrenaline) transporter. Many pheochromocytomas and paragangliomas express norepinephrine (noradrenaline) transporter, as do other NETs (17). Most reports of ${ }^{131} \mathrm{I}-\mathrm{MIBG}$ therapy in patients with pheochromocytoma and paraganglioma are retrospective, small, and use LSA ${ }^{131}$ I-MIBG (Supplemental Table 1; supplemental materials are available at http://jnm. snmjournals.org). No direct therapeutic comparison studies of LSA ${ }^{131}$ I-MIBG and HSA ${ }^{131}$ I-MIBG have been performed.

\section{${ }^{131}$ I-MIBG Production}

LSA ${ }^{131} \mathrm{I}-\mathrm{MIBG}$ preparations $(0.555-1.85 \mathrm{MBq} / \mathrm{mg})$ typically contain approximately $1 / 2,000$ MIBG molecules labeled with ${ }^{131} \mathrm{I}$. Because uptake via norepinephrine (noradrenaline) transporter is saturable, a high administered mass of unlabeled MIBG could competitively inhibit uptake of the ${ }^{131} \mathrm{I}-\mathrm{MIBG}$, potentially reducing therapeutic efficacy, as has been demonstrated in vitro and in preclinical studies (18). Additionally, because MIBG is a competitive inhibitor of catecholamine reuptake, a high administered mass of MIBG can cause pharmacologic effects (19). This is rarely an 
issue for diagnostic administrations (low mass) but occurs more frequently during therapeutic administration of LSA ${ }^{131}$ I-MIBG (high mass).

In contrast, most MIBG molecules are labeled with ${ }^{131} \mathrm{I}$ in preparations of HSA ${ }^{131} \mathrm{I}-\mathrm{MIBG}(92.5 \mathrm{GBq} / \mathrm{mg})$. HSA ${ }^{131} \mathrm{I}-\mathrm{MIBG}$ (Azedra; Progenics Pharmaceuticals, Inc.) was the first radiopharmaceutical the FDA approved to treat metastatic or inoperable pheochromocytoma or paraganglioma $(20,21)$. Use of HSA ${ }^{131} \mathrm{I}-$ MIBG reduces the risk of infusion reactions and may enhance the therapeutic ratio $(19,21,22)$, although the latter has not yet been proven in patients (23).

\section{Patient Selection and Preparation for ${ }^{131}$ I-MIBG RPT}

Published procedure guidelines for ${ }^{131} \mathrm{I}-\mathrm{MIBG}$ therapy preceded availability of HSA ${ }^{131} \mathrm{I}$-MIBG. Nonetheless, these guidelines are broadly applicable to HSA ${ }^{131}$ I-MIBG (24). When MIBG therapy is being considered, a positive ${ }^{123} \mathrm{I}-\mathrm{MIBG}$ scan is required, as only patients with MIBG-avid disease are candidates for therapy. In our opinion, a ${ }^{123} \mathrm{I}-\mathrm{MIBG}$ scan performed within 3-6 mo is adequate if there is no intervening therapy, especially if a ${ }^{131} \mathrm{I}-\mathrm{MIBG}$ dosimetric scan will be performed. The amount of uptake required to allow therapy to be considered is poorly defined. Criteria have included visual assessment (uptake clearly visible above the background level), semiquantitative assessment (lesion-to-background uptake $>2$ ), and estimates of tumor dose or more than $1 \%$ injected dose in tumor (17). Because tumors can lose MIBG avidity over time or after treatment, confirmation of avidity around the time of treatment is important, especially if the patient's symptoms have recently changed. Patients must be screened for marrow function (minimum platelets, $80,000 / \mu \mathrm{L}$; minimum neutrophils, $1,200 / \mu \mathrm{L}$ ) and renal function (minimum creatinine clearance, 30 $\mathrm{mL} / \mathrm{min}$ ). To protect the thyroid by preventing uptake of free ${ }^{131} \mathrm{I}$, stable iodine must be administered unless the thyroid is absent or previously ablated. Typically, $130 \mathrm{mg}$ of an oral solution of potassium iodide (SSKI; Avondale Pharmaceuticals) is administered 24-48 $\mathrm{h}$ before therapy and continued for 10-15 d (24).

\section{${ }^{131}$ I-MIBG Administration}

$H S A{ }^{131} I-M I B G$. Since the approval of HSA ${ }^{131}$ I-MIBG by the FDA, there has been a surge of interest in MIBG therapy. The recommended dose is $296 \mathrm{MBq} / \mathrm{kg}$ in patients weighing no more than $62.5 \mathrm{~kg}$ or $18,500 \mathrm{GBq}$ in those over $62.5 \mathrm{~kg}$, given twice at least $90 \mathrm{~d}$ apart (20). With these doses, virtually all patients exceed Nuclear Regulatory Commission limits for outpatient treatment. Inpatients must be confined to rooms with appropriate shielding, and preparations must be made to limit exposure of other people to the radiation (in the United States, this is usually for 3 nights).

At dosimetric doses of less than $222 \mathrm{MBq}$, HSA ${ }^{131} \mathrm{I}-\mathrm{MIBG}$ can be injected over $1 \mathrm{~min}$, whereas therapeutic doses are administered over $30 \mathrm{~min}$ in adults and $60 \mathrm{~min}$ in children. Representative images for dosimetry and posttreatment HSA ${ }^{131}$ I-MIBG are shown in Figure 1. Occasionally, there is a transient burning pain in the infusion vein, resolving almost immediately with saline flushing.

$L S A{ }^{131} I-M I B G$. In contrast to the 2-injection regimen recommended for HSA ${ }^{131}$ I-MIBG, various approaches using different activities and cycles of LSA ${ }^{131}$ I-MIBG have been reported (Supplemental Table 1) (17). Most inject approximately $7.4 \mathrm{GBq}$ (25-28); 1 group injected approximately $18.5 \mathrm{GBq}$ (29,30). The University of California San Francisco group has used a much higher activity, up to $666 \mathrm{MBq} / \mathrm{kg}$, in conjunction with stem cell support. In the only direct comparison of a very low activity $(5.55$

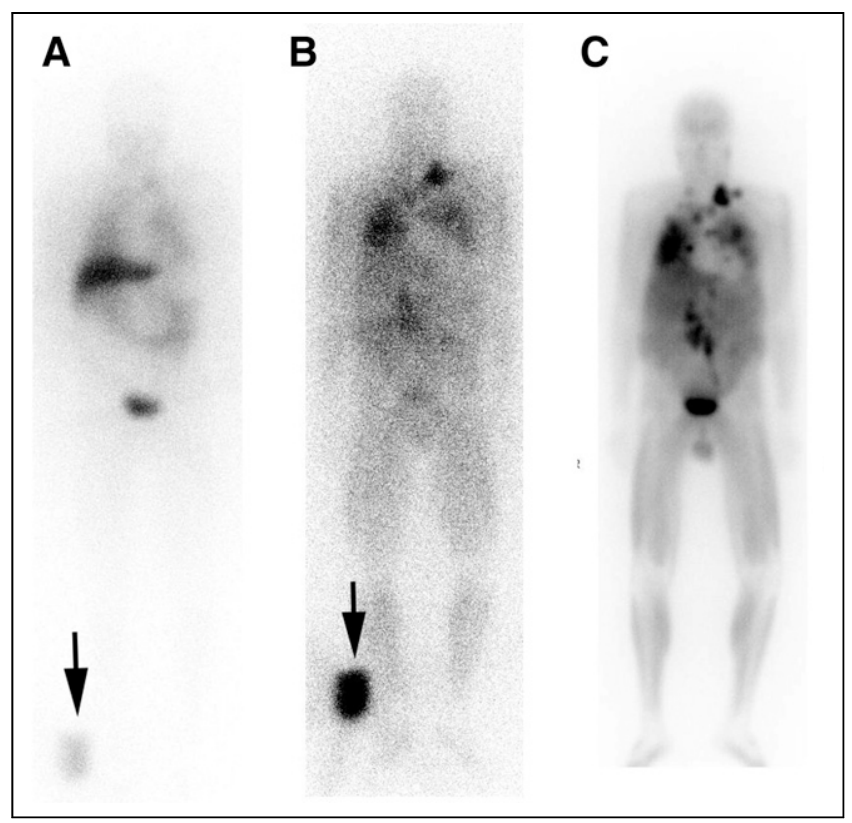

FIGURE 1. Anterior HSA ${ }^{131} \mathrm{I}-\mathrm{MIBG}$ images in 60 -y-old man with metastatic paraganglioma. (A and B) Dosimetric images were performed $1 \mathrm{~h}(\mathrm{~A})$ and $96 \mathrm{~h}(\mathrm{~B})$ after intravenous administration of $185 \mathrm{MBq}$ of HSA ${ }^{131} \mathrm{I}-$ MIBG with standard in field of view (arrows) to allow calculation of organ dose limits. Images show multiple metastases that increase in contrast over time. (C) Three days after therapy with $18.4 \mathrm{GBq}$ of HSA ${ }^{131} \mathrm{I}-\mathrm{MIBG}$, imaging revealed robust retention in sites of disease, including some not visible on dosimetric images. Patient exhibited markedly reduced hormonal symptoms, including decreased need for antihypertensive medications lasting over $2 \mathrm{y}$.

$\mathrm{GBq})$ versus a low-to-intermediate activity $(9.25-12.95 \mathrm{GBq})$, the low-to-intermediate activity had a more rapid onset of efficacy at the expense of increased acute and chronic toxicity (31). Representative diagnostic ${ }^{123}$ I-MIBG images and post-LSA ${ }^{131}$ I-MIBG images are shown in Figure 2. Others have suggested an improved response with higher single administered injections of more than 18.5 GBq versus less than $18.5 \mathrm{GBq}(29)$.

Although high-activity therapies of up to $666 \mathrm{MBq} / \mathrm{kg}$ are common in children with neuroblastoma in conjunction with autologous stem cell support, an at least $444 \mathrm{MBq} / \mathrm{kg}$ activity of LSA ${ }^{131}$ I-MIBG in adults with pheochromocytoma or paraganglioma appears to have high rates of toxicity, particularly pulmonary (15). Therefore, doses higher than $296 \mathrm{MBq} / \mathrm{kg}$ are not recommended.

In contrast to HSA ${ }^{131}$ I-MIBG treatment $(\sim 18.5 \mathrm{GBq})$, lowactivity treatments $(\leq 9.25 \mathrm{GBq})$ can be administered to most patients as outpatients. Therefore, in patients who have relatively indolent disease or who are unwilling to undergo inpatient therapy, serial lowdose treatments can be considered. The most common approaches to low-dose therapy are $74-111 \mathrm{MBq} / \mathrm{kg}$ or $7.40 \mathrm{GBq} /$ cycle administered 3 mo apart. Many practitioners give 3-4 cycles of therapy followed by reassessment and consideration of further therapy.

Because LSA ${ }^{131}$ I-MIBG treatments contain a much higher mass of MIBG than do HSA ${ }^{131}$ I-MIBG treatments, the former are usually administered over $1-2 \mathrm{~h}(15,17,25,27,32)$, although shorter infusions have been performed (26,30). Unlike HSA ${ }^{131} \mathrm{I}-\mathrm{MIBG}$, which is not associated with hypertension during infusion, hypertension occurs during $6 \%-14 \%$ of LSA ${ }^{131}$ I-MIBG infusions, sometimes requiring pausing or decreasing the infusion rate and using antihypertensive treatment $(15,21,33,34)$. Nonetheless, 


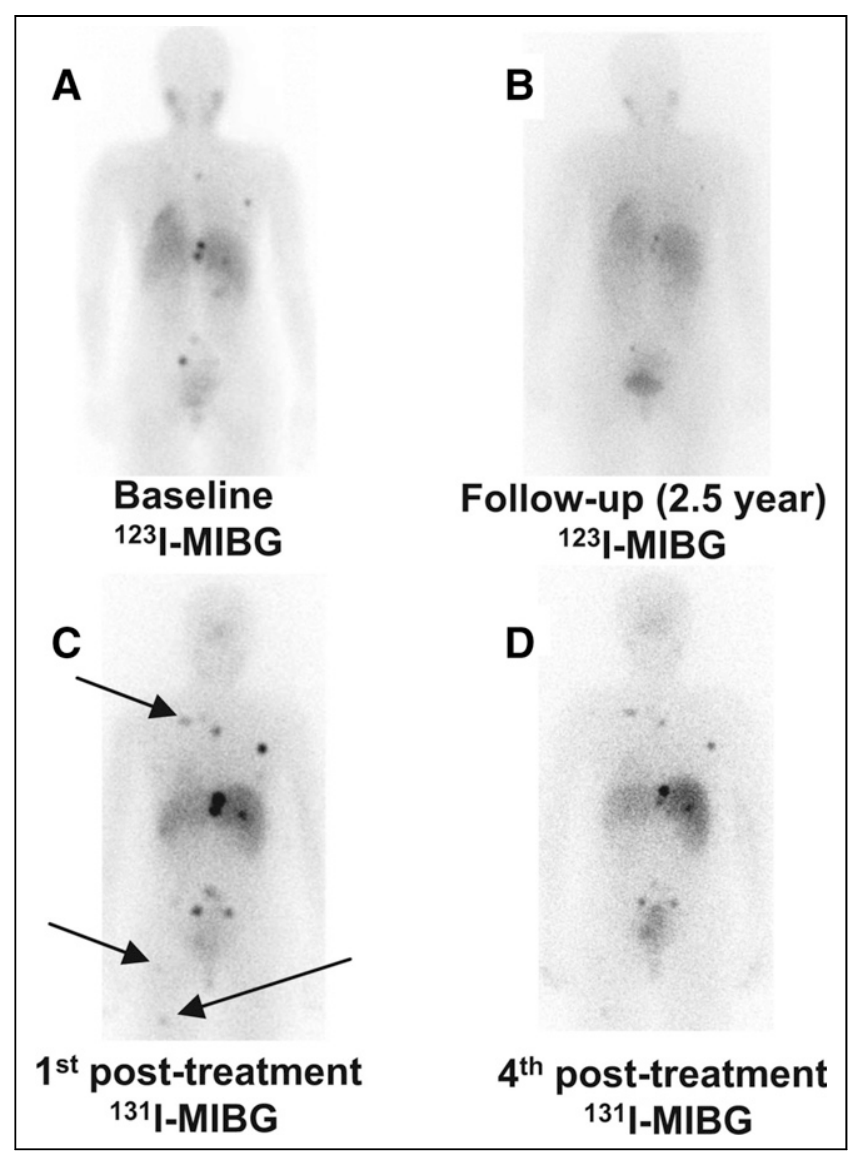

FIGURE 2. Patient with metastatic pheochromocytoma to bone with bone pain. Patient received 4 treatments of LSA ${ }^{131} \mathrm{I}-\mathrm{MIBG}(111 \mathrm{MBq} / \mathrm{kg}$; range, 5.74-6.18 GBq per treatment) totaling $24.01 \mathrm{GBq}$ over $14 \mathrm{mo}$. Patient showed symptomatic improvement in bone pain. ${ }^{123} \mathrm{I}-\mathrm{MIBG}$ scans were obtained at baseline $(\mathrm{A})$ and at $2.5 \mathrm{y}(\mathrm{B})$ after last ${ }^{131} \mathrm{I}$-MIBG treatment. (C) ${ }^{131}$ I-MIBG posttherapy scan a few days after first LSA ${ }^{131}$ I-MIBG treatment shows better visualization of lesion, and more lesions are evident (arrows) than on baseline ${ }^{123}$ I-MIBG scan (A). (D) ${ }^{123}$ I-MIBG scan after all treatments (B) and last ${ }^{131} \mathrm{I}$-MIBG posttreatment scan (D) shows decreased uptake in bone lesions, probably related to treatment. (Reprinted with permission of (66).)

significant worsening of preexisting hypertension in the first 24 $\mathrm{h}$ is reported in $11 \%$ of patients receiving HSA ${ }^{131} \mathrm{I}-\mathrm{MIBG}$ and may also occur with LSA ${ }^{131} \mathrm{I}$-MIBG.

\section{Clinical Experience with ${ }^{131} \mathrm{I}-\mathrm{MIBG}$ in Pheochromocytoma and Paraganglioma}

There have been few controlled prospective trials and no phase III clinical trials of ${ }^{131}$ I MIBG therapy. Loh et al. reviewed the experience of 21 centers using LSA ${ }^{131}$ I-MIBG receiving an average of 3 (range, 1-11) single injections of 3.55-11.1 GBq and cumulative activities of 3.55-85.91 GBq (35). Thirty percent of patients had at least partial responses, and $4 \%$ had complete responses. Furthermore, there was at least a partial hormonal response in $45 \%$ of patients, $13 \%$ of whom had a complete response. A metanalysis that included 243 patients with metastatic pheochromocytoma or paraganglioma receiving median cumulative activities ranging from $6.88-39.4 \mathrm{GBq}$ with a median of 1-7 infusions revealed similar findings (36). Supplemental Table 1 shows therapeutic reports with 20 or more patients, including 2 reports using HSA ${ }^{131} \mathrm{I}-\mathrm{MIBG}$.
The pivotal prospective phase II trial supporting FDA approval of HSA ${ }^{131}$ I-MIBG (21) enrolled 68 patients with advanced pheochromocytoma or paraganglioma and disease-related hypertension. For LSA ${ }^{131}$ I-MIBG, the largest prospective trial on pheochromocytoma and paraganglioma enrolled 50 subjects treated with varying activities of LSA ${ }^{131} \mathrm{I}-\mathrm{MIBG}$, including some who received myeloablative doses and stem cell support (15). Using RECIST, both trials found a high rate of partial or durable stable disease in $92 \%$ of patients. However, an objective tumor shrinkage meeting RECIST for partial response was seen in only $22 \%-23 \%$ of patients. Furthermore, the peak anatomic response was often delayed, taking up to 12 mo from the first treatment. Other studies have shown somewhat higher objective response rates $(34 \%-38 \%)$ using single administrations of approximately $14.467-18.5 \mathrm{GBq}(29,30)$. At lower injected activities, objective response rates of $25 \%-47 \%$ were observed $(26,27,31,37)$. In general, studies using lower single administered activities usually included a larger number of treatment cycles.

Biochemically, $31 \%-35 \%$ of the HSA ${ }^{131}$ I-MIBG group had a response in norepinephrine and normetanephrine levels, and $68 \%$ of patients had a reduced chromogranin A level. In comparison, therapy with LSA ${ }^{131}$ I-MIBG resulted in a complete or partial catecholamine or metanephrine response in $19 \%-100 \%$ of patients (Supplemental Table 1).

The endpoint of the HSA ${ }^{131}$ I-MIBG study by Pryma et al. was blood pressure control; $68 \%$ of subjects had at least a $50 \%$ reduction in antihypertensive medication use lasting at least 6 mo (38). Although no studies of LSA ${ }^{131}$ I-MIBG focused on hypertension control, a report by Thorpe et al. described improvement in $14 \%$ of hypertensive patients (30). Response in terms of overall survival and progression-free survival (Supplemental Table 1) has been variable, with some reporting progression-free survival of at least $2 \mathrm{y}(25,30)$ and median overall survival of more than $3 \mathrm{y}$ $(21,29,30,39)$.

In patients with soft-tissue disease, the response to RPT is typically evaluated using anatomic imaging, most commonly CT scans. Other investigators have used functional imaging, including ${ }^{123} \mathrm{I}-\mathrm{MIBG}$, but given the quantitative nature of PET in patients with ${ }^{18}$ F-FDG-avid disease, ${ }^{18}$ F-FDG has also been used for response evaluation.

\section{Dosimetric Scans}

The HSA ${ }^{131}$ I-MIBG regimen incorporates an initial dosimetric study using HSA ${ }^{131} \mathrm{I}$-MIBG $(3.7 \mathrm{MBq} / \mathrm{kg}$, not to exceed 222 $\mathrm{MBq}$ ), with whole-body imaging on the day of injection, 1-2 d afterward, and 2-5 d afterward, using the MIRD schema to ensure that delivered doses are within specified limits. Usually, the doselimiting factor is renal exposure; any required activity reduction is split equally across the 2 planned treatments.

\section{Side Effects and Toxicity Profile}

Toxicity with ${ }^{131}$ I-MIBG therapy is common; most patients recover with conservative care. The most common adverse reactions with HSA ${ }^{131}$ I-MIBG are hematologic, with grade 3-4 hematologic toxicity in about $40 \%$ of patients treated with $296 \mathrm{MBq} / \mathrm{kg}$, increasing to over $80 \%$ at higher doses $(\sim 444 \mathrm{MBq} / \mathrm{kg})$. At the lower dose, $25 \%$ of patients required some hematologic support (most commonly packed red blood cells); careful monitoring of hematologic parameters after therapy is critical. Most patients reach nadir 4-6 wk after therapy, but monitoring should start no later than 2 wk after therapy. The risk of dose-limiting toxicity increases with retreatment, but because efficacy appears to remain 
strong, particularly in those with favorable prior responses, patients with an adequate marrow reserve can safely be treated again. The incidence of myelodysplasia and leukemia after HSA ${ }^{131} \mathrm{I}-\mathrm{MIBG}$ is $6.8 \%$, similar to the $3.9 \%-7 \%$ reported with LSA ${ }^{131} \mathrm{I}-\mathrm{MIBG}(15,31)$.

A $6.8 \%$ rate of hypothyroidism is noted with HSA ${ }^{131} \mathrm{I}-\mathrm{MIBG}$, and $11 \%-20 \%$ with LSA ${ }^{131}$ I-MIBG $(25,39,40)$, despite attempts at thyroid blockade. Gastrointestinal toxicity is common, with nausea and vomiting in $50 \%-75 \%$ of patients treated with a 296 $\mathrm{MBq} / \mathrm{kg}$ dose of HSA ${ }^{131} \mathrm{I}-\mathrm{MIBG}$; results are anecdotally similar with LSA ${ }^{131}$ I-MIBG. However, because grade 3 or greater gastrointestinal toxicity is extremely rare, conservative care with antiemetics and hydration is almost always adequate.

Most adverse effects were seen in heavily pretreated patients. The toxicity profile in less heavily pretreated patients remains to be seen.

In general, trials describing low-dose therapy (e.g., $74 \mathrm{MBq} / \mathrm{kg}$ ) have similar but less severe toxicity, with lower (but nonzero) rates of grade 3-4 toxicity. However, hematologic toxicity remains common, particularly in later cycles of therapy, and careful monitoring is still indicated.

\section{Summary}

MIBG scintigraphy is the first step to determining eligibility for ${ }^{131}$ I-MIBG therapy. Given the overlapping therapeutic responses of LSA ${ }^{131}$ I-MIBG and HSA ${ }^{131}$ I-MIBG, and that only HSA ${ }^{131} \mathrm{I}$ MIBG is FDA-approved, HSA ${ }^{131} \mathrm{I}-\mathrm{MIBG}$ is recommended in the United States. Nonetheless, considering other factors such as the cost and whether the therapy will be inpatient or outpatient, lowactivity treatment strategies may be preferred, especially outside the United States. Future studies are needed to guide clinicians on choosing the MIBG treatment regimen most suited to their patients' needs, including whether administration of a low activity and more frequent cycles of treatment with HSA ${ }^{131}$ I-MIBG would be useful. These trials should consider not only efficacy but also other parameters such as toxicity, side effects, and patient preferences.

\section{PRRT WITH RADIOLABELED SOMATOSTATIN ANALOGS}

Somatostatin receptors are highly expressed in NETs and have been targets for imaging and therapy, as reviewed recently in our continuing education article on imaging pheochromocytoma and paraganglioma (3). Somatostatin is a natural 14-amino-acid peptide hormone with regulatory effects in the endocrine system via binding to somatostatin receptors $1-5$, which are highly expressed in NETs. Several somatostatin receptor agonists have been developed for imaging, and ${ }^{177} \mathrm{Lu}$-DOTATATE is now available for therapeutic purposes. At present, this is the only PRRT agent approved by the FDA and the European Medicines Agency. The North American Neuroendocrine Tumor Society and Society of Nuclear Medicine and Molecular Imaging jointly published guidelines addressing screening, preparation, administration, radiation safety, adverse events monitoring, and follow-up for ${ }^{177} \mathrm{Lu}-$ DOTATATE treatment (41). Although pheochromocytoma or paraganglioma is not an approved indication for ${ }^{177}$ Lu-DOTATATE, National Comprehensive Cancer Network guidelines provide a rationale for its off-label use (42).

\section{Patient Selection and Preparation}

A prerequisite for PRRT therapy of pheochromocytoma and paraganglioma is tumor avidity on somatostatin receptor imaging.
This avidity was initially determined with ${ }^{111}$ In-pentetreotide using a qualitative visual measure of uptake known as the Krenning score (43). The Krenning score uses a 4-point scale in which grade 1 indicates uptake less than in the liver; grade 2, uptake equal to that in the liver; grade 3, uptake greater than in the liver; and grade 4, uptake greater than in the spleen or kidneys. Generally, tumors should have a score of grade 2 or higher to be eligible for PRRT. Almost universally, tumors appear more prominent on ${ }^{68}$ Ga-DOTATATE PET (43); nonetheless, the same scoring concept is used, although significant differences from ${ }^{111}$ In-pentetreotide scans can be present.

Because the dose-limiting organs in PRRT are the kidneys (44), it is desirable to have normal renal function (glomerular filtration rate $>50 \mathrm{~mL} / \mathrm{h}$ ) before treatment. Furthermore, adequate liver and bone marrow function should be documented, with the typical parameters being a hemoglobin level higher than $8 \mathrm{~g} / \mathrm{dL}$, a white blood cell count higher than $2.0 \mathrm{~K} / \mu \mathrm{L}$, a platelet count higher than $70 \mathrm{~K} / \mu \mathrm{L}$, and a total bilirubin level lower than 3 times the upper limit of normal (41).

Most guidelines recommend stopping short- and long-acting octreotide for at least $24 \mathrm{~h}$ and 3-4 wk, respectively, before ${ }^{90} \mathrm{Y}$ or ${ }^{177} \mathrm{Lu}$-DOTA-somatostatin analog administration because of the risk of competition for, or blocking of, uptake. However, recent studies indicate that octreotide administration does not decrease tumor accumulation of ${ }^{68} \mathrm{Ga}$-DOTATATE, although decreases in normal spleen, liver, and thyroid uptake were observed $(45,46)$.

\section{PRRT Administration}

Early ${ }^{90}$ Y-DOTATOC studies showed cases of acute renal failure related to reabsorption and retention of the agent by the proximal renal tubules (44). However, administration of amino acid solutions containing lysine and arginine blocked renal uptake by approximately $40 \%(47,48)$. Although initial protocols used commercially available amino acid mixtures such as Clinisol (Baxter International Inc.) and Aminosyn II (Abbott Laboratories), solutions containing only lysine and arginine (18-25 g total of each, $1-2 \mathrm{~L}$, osmolality $\leq 1,050 \mathrm{mOsmol})$ minimize nausea and vomiting and are now preferred (41). Patients are pretreated with antiemetics followed by a continuous infusion of amino acids starting $30 \mathrm{~min}$ before ${ }^{177} \mathrm{Lu}$-DOTATATE and continued during and after treatment for a total of approximately $4 \mathrm{~h}$ until the entire solution has been infused.

Measured exposure rates from patients treated with 7.4 GBq of ${ }^{177} \mathrm{Lu}$-DOTATATE are low (under $4 \mathrm{mR} / \mathrm{h}$ at $1 \mathrm{~min}$ ), resulting in doses to the public and caregivers of less than 0.5 cGy per year, thus allowing for outpatient treatment in the United States (49).

\section{Clinical Experience with PRRT in Pheochromocytoma and Paraganglioma \\ ${ }^{90}$ Y-DOTATOC and ${ }^{90}$ Y-DOTATATE are not commercially} available, and their use is limited to certain academic centers. In contrast, ${ }^{177}$ Lu-DOTATATE has been approved as safe and effective in gastroenteropancreatic NETs. In pheochromocytoma and paraganglioma, small studies have also shown benefit with limited toxicity.

Supplemental Table 2 shows studies containing 5 or more patients receiving a ${ }^{90} \mathrm{Y}$ - or ${ }^{177} \mathrm{Lu}$-DOTA-somatostatin analog for metastatic or inoperable pheochromocytoma or paraganglioma. A metaanalysis found similar response rates between ${ }^{90}$ Y-DOTATOC and ${ }^{177} \mathrm{Lu}-$ DOTATATE, with $25 \%$ of patients demonstrating an objective response; 61\%, a clinical response; and 84\%, biochemical 


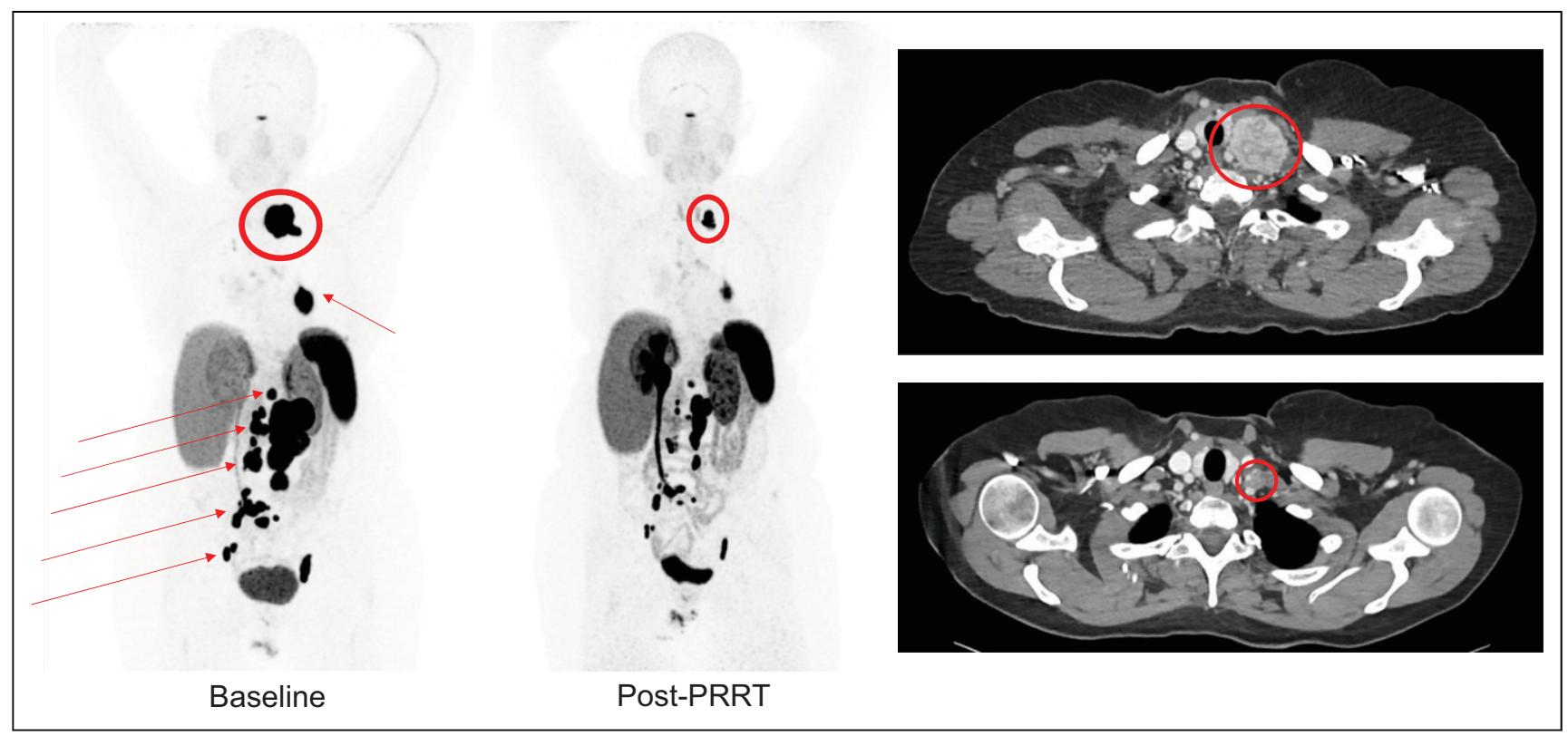

FIGURE 3. A 53-y-old woman with metastatic hormone-secreting SDHB-associated pheochromocytoma. Maximal-intensity-projection images of ${ }^{68} \mathrm{Ga}$-DOTATATE at baseline (left) vs. $12 \mathrm{mo}$ after PRRT with $7.4 \mathrm{GBq}$ of ${ }^{177}$ Lu-DOTATATE 4 times (middle) demonstrate significant decrease in tracer uptake in tumors in neck (encircled), left hilum, retroperitoneum, and pelvis (arrows). Baseline axial CT image (top right) through neck demonstrates large left supraclavicular mass at baseline (encircled), and post-PRRT axial CT image (bottom right) shows significant decrease in tumor size (encircled).

improvement. Estimated overall survival was $54.5 \mathrm{mo}$, and mean progression-free survival was 37.1 mo (50). Reports using ${ }^{177} \mathrm{Lu}-$ DOTATATE have demonstrated significant partial regressions and stable disease as determined by anatomic imaging, as well as significant biochemical and symptomatic responses (Supplemental Table 2). Representative examples of pre- and post-PRRT ${ }^{68} \mathrm{Ga}$-DOTATATE and CT images are shown in Figure 3.

\section{Dosimetry}

The target organs for PRRT toxicity are the kidneys and bone marrow, as has been defined for nonpheochromocytoma and nonparaganglioma NETs. Bone marrow toxicity with a 50\% drop in platelet counts is associated with a marrow dose of 2 Gy $(51,52)$. Although a limit of 23-Gy cumulative absorbed dose can be used to avoid toxicity to the kidneys with biologically effective dose determinations, these limits could be better defined (52). Furthermore, other defined renal limits for patients with risk factors $(28 \mathrm{~Gy})$ may be lower than for those without risk factors (40 Gy) (53).

At present, we recommend that $7.4 \mathrm{GBq}$ every 8 wk for 4 cycles, as approved by the FDA for gastroenteropancreatic NETs, also be used in patients with metastatic or inoperable pheochromocytoma or paraganglioma. Although there are no requirements for dosimetry, it should be considered if higher activities are administered. Groups that treat with more than $7.4 \mathrm{GBq} /$ cycle or more than 4 cycles use dosimetry to limit the radiation dose to 23 Gy to the kidneys and less than 2 Gy to the bone marrow and have been able to administer up to 11 cycles with a maximum cumulative activity of $81.4 \mathrm{GBq}$ versus the $29.6 \mathrm{GBq}$ recommended in the package insert (54).

\section{Response Evaluation with PRRT}

Evaluating response to PRRT can be challenging. Morphologic criteria such as RECIST 1.1 are the most frequently used, but anatomic changes often lag behind functional changes in pheochromocytoma and paraganglioma and may be better detected using ${ }^{68}$ Ga-DOTATATE, ${ }^{18} \mathrm{~F}-\mathrm{FDG}$, or other functional PET radiopharmaceuticals (55). Another issue is pseudoprogression, in which $9 \%$ of patients with RECIST-stable disease evidenced transient increases in tumor size by more than $10 \%$ at $6 \mathrm{wk}$ after treatment (50). Three months appears to be the optimal time to determine treatment response. Changes in clinical status should guide the timing and interval of reevaluation.

\section{Side Effects and Toxicity Profile}

Although the toxicity profiles for ${ }^{90} \mathrm{Y}$ - and ${ }^{177} \mathrm{Lu}-\mathrm{PRRT}$ have been characterized in patients with NETs, we feel that the toxicity profile in pheochromocytoma and paraganglioma is similar (57). Nonetheless, patients with pheochromocytoma or paraganglioma are at higher risk for certain side effects such as catecholamine release syndrome and tumor lysis syndrome $(13,14)$.

A long-term follow-up study of patients receiving ${ }^{90} \mathrm{Y}$-DOTATOC versus ${ }^{177} \mathrm{Lu}$-DOTATATE with nephroprotection in NET found a slightly greater drop in renal function over time for ${ }^{90} \mathrm{Y}$ DOTATOC than for ${ }^{177}$ Lu-DOTATATE (58), although the overall incidence was low. In the pivotal NETTER-1 trial of ${ }^{177} \mathrm{Lu}-$ DOTATATE in gastroenteropancreatic NET, renal failure of all grades occurred in $12 \%$ of patients, with $3 \%$ having grade 3 or 4 toxicity. In pheochromocytoma and paraganglioma reports, approximately $9 \%$ had renal toxicity higher than grade 3 (59).

Hematologic side effects are usually mild. The incidence of anemia in the NETTER-1 trial was $81 \%$ overall, with no grade 3-4 events. Thrombocytopenia and neutropenia of any grade occurred in $53 \%$ and $26 \%$ of patients, with grade $3-4$ events in $1 \%$ and $3 \%$, respectively. Grade 3 and 4 decreases in lymphocytes are frequent but do not require dose modifications (60). However, myelodysplastic syndrome or acute leukemia was reported in $3.4 \%$ of patients with nonpheochromocytoma or nonparaganglioma (61). In patients with pheochromocytoma or paraganglioma treated with PRRT, the incidence of myelodysplasia is similarly low, ranging from $2.5 \%$ to $8.3 \%(14,62,63)$. 


\section{Summary}

The available retrospective data considering PRRT in metastatic or inoperable pheochromocytoma and paraganglioma show promise. Although complete responses as defined by RECIST have not been reported, a significant number of patients are able to achieve partial responses, and many have stable disease. Side effects are generally mild and well tolerated. An ongoing prospective phase II clinical trial (NCT03206060) of patients with sporadic or SDHx-related metastatic or inoperable pheochromocytoma or paraganglioma with clear evidence of progression receiving $7.4 \mathrm{GBq}$ of ${ }^{177} \mathrm{Lu}$-DOTATATE every $8 \mathrm{wk}$ ( 4 cycles) is ongoing. Preliminary evaluation after 2 cycles of treatment using functional imaging in 11 patients has shown promising results (64). Until final results are available, the National Comprehensive Cancer Network recommendations indicate that the use of ${ }^{177}$ Lu-DOTATATE with the activity and schedule stated in the package insert may be beneficial to many patients with metastatic or inoperable pheochromocytoma or paraganglioma.

If tumor uptake of ${ }^{177} \mathrm{Lu}-\mathrm{DOTATATE}$ and ${ }^{131} \mathrm{I}-\mathrm{MIBG}$ are equally good, the selection of radiopharmaceutical for RPT will rely on other considerations (65).

\section{CONCLUSION}

Currently, ${ }^{131} \mathrm{I}-\mathrm{MIBG}$ and PRRT are 2 RPT approaches that have shown efficacy in patients with metastatic or inoperable pheochromocytoma or paraganglioma, with acceptable toxicity profiles. Once the decision to use RPT is made, one must decide which of these 2 approaches to pursue. The first consideration is based on the degree of tumor localization of radiolabeled MIBG or DOTA-somatostatin analog. Given that disparate imaging results are not uncommon, this consideration will often inform the selection. However, if both tracers demonstrate good localization, other considerations, including cost, whether the treatment is inpatient or outpatient, and preexisting organ toxicities such as renal abnormalities or marrow toxicity, must be considered to pair each patient with the optimal radiopharmaceutical.

\section{REFERENCES}

1. Berends AMA, Buitenwerf E, de Krijger RR, et al. Incidence of pheochromocytoma and sympathetic paraganglioma in The Netherlands: a nationwide study and systematic review. Eur J Intern Med. 2018;51:68-73.

2. Lam AK. Update on adrenal tumours in 2017 World Health Organization (WHO) of endocrine tumours. Endocr Pathol. 2017;28:213-227.

3. Carrasquillo JA, Chen CC, Jha A, et al. Imaging of pheochromocytoma and paraganglioma. J Nucl Med. 2021;62:1033-1042.

4. Turkova H, Prodanov T, Maly M, et al. Characteristics and outcomes of metastatic SDHB and sporadic pheochromocytoma/paraganglioma: an National Institutes of Health study. Endocr Pract. 2016;22:302-314.

5. Sisson JC, Shulkin BL, Esfandiari NH. Courses of malignant pheochromocytoma: implications for therapy. Ann N Y Acad Sci. 2006;1073:505-511.

6. Hescot S, Curras-Freixes M, Deutschbein T, et al. Prognosis of malignant pheochromocytoma and paraganglioma (MAPP-Prono Study): A European Network for the Study of Adrenal Tumors retrospective study. J Clin Endocrinol Metab. 2019;104:2367-2374.

7. Crona J, Lamarca A, Ghosal S, Welin S, Skogseid B, Pacak K. Genotype-phenotype correlations in pheochromocytoma and paraganglioma: a systematic review and individual patient meta-analysis. Endocr Relat Cancer. 2019;26:539-550.

8. Hescot S, Leboulleux S, Amar L, et al. One-year progression-free survival of therapy-naive patients with malignant pheochromocytoma and paraganglioma. J Clin Endocrinol Metab. 2013;98:4006-4012.

9. Niemeijer ND, Alblas G, van Hulsteijn LT, Dekkers OM, Corssmit EP. Chemotherapy with cyclophosphamide, vincristine and dacarbazine for malignant paraganglioma and pheochromocytoma: systematic review and meta-analysis. Clin Endocrinol (Oxf). 2014;81:642-651.
10. O'Kane GM, Ezzat S, Joshua AM, et al. A phase 2 trial of sunitinib in patients with progressive paraganglioma or pheochromocytoma: the SNIPP trial. $\mathrm{Br} J$ Cancer. 2019;120:1113-1119.

11. Vogel J, Atanacio AS, Prodanov T, et al. External beam radiation therapy in treatment of malignant pheochromocytoma and paraganglioma. Front Oncol. 2014;4:166.

12. Pacak K. Preoperative management of the pheochromocytoma patient. J Clin Endocrinol Metab. 2007;92:4069-4079.

13. Makis W, McCann K, McEwan AJ. The challenges of treating paraganglioma patients with ${ }^{177} \mathrm{Lu}$-DOTATATE PRRT: catecholamine crises, tumor lysis syndrome and the need for modification of treatment protocols. Nucl Med Mol Imaging. 2015;49:223-230.

14. Zandee WT, Feelders RA, Smit Duijzentkunst DA, et al. Treatment of inoperable or metastatic paragangliomas and pheochromocytomas with peptide receptor radionuclide therapy using ${ }^{177}$ Lu-DOTATATE. Eur J Endocrinol. 2019;181:45-53.

15. Gonias S, Goldsby R, Matthay KK, et al. Phase II study of high-dose [ $\left.{ }^{131} \mathrm{I}\right]$ metaiodobenzylguanidine therapy for patients with metastatic pheochromocytoma and paraganglioma. J Clin Oncol. 2009;27:4162-4168.

16. Wieland DM, Wu JI, Brown LE, Mangner TJ, Swanson DP, Beierwaltes WH. Radiolabeled adrenergic neuron-blocking agents - adrenomedullary imaging with $\left[{ }^{131}\right.$ I]iodobenzylguanidine. J Nucl Med. 1980;21:349-353.

17. Carrasquillo JA, Pandit-Taskar N, Chen CC. Radionuclide therapy of adrenal tumors. J Surg Oncol. 2012;106:632-642.

18. Mairs RJ, Russell J, Cunningham S, et al. Enhanced tumor uptake and in-vitro radiotoxicity of no-carrier-added $\left[{ }^{131} \mathrm{I}\right]$ metaiodobenzylguanidine: implications for the targeted radiotherapy of neuroblastoma. Eur J Cancer. 1995;31A:576-581.

19. Barrett JA, Joyal JL, Hillier SM, et al. Comparison of high-specific-activity Ultratrace ${ }^{123} /{ }^{131}$ I-MIBG and carrier-added ${ }^{123} /{ }^{131}$ I-MIBG on efficacy, pharmacokinetics, and tissue distribution. Cancer Biother Radiopharm. 2010;25:299-308.

20. Noto RB, Pryma DA, Jensen J, et al. Phase 1 study of high-specific-activity I-131 MIBG for metastatic and/or recurrent pheochromocytoma or paraganglioma. J Clin Endocrinol Metab. 2018;103:213-220.

21. Pryma DA, Chin BB, Noto RB, et al. Efficacy and safety of high-specific-activity ${ }^{131}$ I-MIBG therapy in patients with advanced pheochromocytoma or paraganglioma. J Nucl Med. 2019;60:623-630.

22. Coleman RE, Stubbs JB, Barrett JA, de la Guardia M, Lafrance N, Babich JW. Radiation dosimetry, pharmacokinetics, and safety of Ultratrace iobenguane I-131 in patients with malignant pheochromocytoma/paraganglioma or metastatic carcinoid. Cancer Biother Radiopharm. 2009;24:469-475.

23. Owens J, Bolster AA, Prosser JE, et al. No-carrier-added ${ }^{123} \mathrm{I}-\mathrm{MIBG}$ : an initial clinical study in patients with phaeochromocytoma. Nucl Med Commun. 2000;21:437-440.

24. Giammarile F, Chiti A, Lassmann M, Brans B, Flux G. EANM. EANM procedure guidelines for ${ }^{131} \mathrm{I}$-meta-iodobenzylguanidine $\left({ }^{131} \mathrm{I}-\mathrm{mIBG}\right)$ therapy. Eur $\mathrm{J}$ Nucl Med Mol Imaging. 2008;35:1039-1047.

25. Shapiro B, Sisson IC, Wieland DM, et al. Radiopharmaceutical therapy of malignant pheochromocytoma with $\left[{ }^{131} \mathrm{I}\right]$ metaiodobenzylguanidine: results from ten years of experience. J Nucl Biol Med. 1991;35:269-276.

26. Mukherjee JJ, Kaltsas GA, Islam N, et al. Treatment of metastatic carcinoid tumours, phaeochromocytoma, paraganglioma and medullary carcinoma of the thyroid with ${ }^{131}$ I-meta-iodobenzylguanidine ${ }^{[131} \mathrm{I}-\mathrm{mIBG}$. Clin Endocrinol (Oxf). 2001;55:47-60.

27. Gedik GK, Hoefnagel CA, Bais E, Olmos RA. ${ }^{131} \mathrm{I}-\mathrm{MIBG}$ therapy in metastatic phaeochromocytoma and paraganglioma. Eur J Nucl Med Mol Imaging. 2008;35:725-733.

28. Wakabayashi H, Taki J, Inaki A, et al. Prognostic values of initial responses to low-dose ${ }^{131}$ I-MIBG therapy in patients with malignant pheochromocytoma and paraganglioma. Ann Nucl Med. 2013;27:839-846.

29. Safford SD, Coleman RE, Gockerman JP, et al. Iodine-131 metaiodobenzylguanidine is an effective treatment for malignant pheochromocytoma and paraganglioma. Surgery. 2003;134:956-962.

30. Thorpe MP, Kane A, Zhu J, Morse MA, Wong T, Borges-Neto S. Long-term outcomes of 125 patients with metastatic pheochromocytoma or paraganglioma treated with 131-I MIBG. J Clin Endocrinol Metab. 2020;105:E494-E501.

31. Castellani MR, Seghezzi S, Chiesa C, et al. ${ }^{131}$ I-MIBG treatment of pheochromocytoma: low versus intermediate activity regimens of therapy. Q J Nucl Med Mol Imaging. 2010;54:100-113.

32. Schlumberger M, Gicquel C, Lumbroso J, et al. Malignant pheochromocytoma: clinical, biological, histologic and therapeutic data in a series of 20 patients with distant metastases. J Endocrinol Invest. 1992;15:631-642.

33. Kotecka-Blicharz A, Hasse-Lazar K, Handkiewicz-Junak D, et al. 131-I MIBG therapy of malignant pheochromocytoma and paraganglioma tumours: a singlecentre study. Endokrynol Pol. 2018;69:246-251. 
34. Yoshinaga K, Oriuchi N, Wakabayashi H, et al. Effects and safety of ${ }^{131}$ I-metaiodobenzylguanidine (MIBG) radiotherapy in malignant neuroendocrine tumors: results from a multicenter observational registry. Endocr J. 2014;61:1171-1180.

35. Loh KC, Fitzgerald PA, Matthay KK, Yeo PP, Price DC. The treatment of malignant pheochromocytoma with iodine-131 metaiodobenzylguanidine ( $\left.{ }^{131} \mathrm{I}-\mathrm{MIBG}\right)$ : a comprehensive review of 116 reported patients. J Endocrinol Invest. 1997;20:648-658.

36. van Hulsteijn LT, Niemeijer ND, Dekkers OM, Corssmit EP. ${ }^{131}$ I-MIBG therapy for malignant paraganglioma and phaeochromocytoma: systematic review and meta-analysis. Clin Endocrinol (Oxf). 2014;80:487-501.

37. Krempf M, Lumbroso J, Mornex R, et al. Use of $\mathrm{m}-\left[{ }^{131} \mathrm{I}\right]$ iodobenzylguanidine in the treatment of malignant pheochromocytoma. $J$ Clin Endocrinol Metab. 1991;72:455-461.

38. Pryma D, Divgi C. Meta-iodobenzyl guanidine for detection and staging of neuroendocrine tumors. Nucl Med Biol. 2008;35(suppl 1):S3-S8.

39. Rutherford MA, Rankin AJ, Yates TM, et al. Management of metastatic phaeochromocytoma and paraganglioma: use of iodine-131-meta-iodobenzylguanidine therapy in a tertiary referral centre. QJM. 2015;108:361-368.

40. Shilkrut M, Bar-Deroma R, Bar-Sela G, Berniger A, Kuten A. Low-dose iodine-131 metaiodobenzylguanidine therapy for patients with malignant pheochromocytoma and paraganglioma: single center experience. Am J Clin Oncol. 2010;33:79-82.

41. Hope TA, Abbott A, Colucci K, et al. NANETS/SNMMI procedure standard for somatostatin receptor-based peptide receptor radionuclide therapy with ${ }^{177} \mathrm{Lu}-$ DOTATATE. J Nucl Med. 2019;60:937-943.

42. Shah MH, Goldner WS, Halfdanarson TR, et al. NCCN guidelines insights: neuroendocrine and adrenal tumors, version 2.2018. J Natl Compr Canc Netw. 2018;16: 693-702.

43. Hope TA, Calais J, Zhang L, Dieckmann W, Millo C. ${ }^{111}$ In-pentetreotide scintigraphy versus ${ }^{68} \mathrm{Ga}$-DOTATATE PET: impact on Krenning scores and effect of tumor burden. J Nucl Med. 2019;60:1266-1269.

44. Otte A, Herrmann R, Heppeler A, et al. Yttrium-90 DOTATOC: first clinical results. Eur J Nucl Med. 1999;26:1439-1447.

45. Aalbersberg EA, de Wit-van der Veen BJ, Versleijen MWJ, et al. Influence of lanreotide on uptake of ${ }^{68} \mathrm{Ga}$-DOTATATE in patients with neuroendocrine tumours: a prospective intra-patient evaluation. Eur J Nucl Med Mol Imaging. 2019;46:696-703.

46. Ayati N, Lee ST, Zakavi R, et al. Long-acting somatostatin analog therapy differentially alters ${ }^{68} \mathrm{Ga}$-DOTATATE uptake in normal tissues compared with primary tumors and metastatic lesions. J Nucl Med. 2018;59:223-227.

47. Hammond PJ, Wade AF, Gwilliam ME, et al. Amino acid infusion blocks renal tubular uptake of an indium-labelled somatostatin analogue. Br J Cancer. 1993;67:1437-1439.

48. Mogensen CE, Sølling K. Studies on renal tubular protein reabsorption: partial and near complete inhibition by certain amino acids. Scand J Clin Lab Invest. 1977;37:477-486.

49. Calais PJ, Turner JH. Radiation safety of outpatient ${ }^{177}$ Lu-octreotate radiopeptide therapy of neuroendocrine tumors. Ann Nucl Med. 2014;28:531-539.

50. Satapathy S, Mittal BR, Bhansali A. Peptide receptor radionuclide therapy in the management of advanced pheochromocytoma and paraganglioma: a systematic review and meta-analysis. Clin Endocrinol (Oxf). 2019;91:718-727.
51. Walrand S, Barone R, Pauwels S, Jamar F. Experimental facts supporting a red marrow uptake due to radiometal transchelation in ${ }^{90}$ Y-DOTATOC therapy and relationship to the decrease of platelet counts. Eur J Nucl Med Mol Imaging. 2011;38:1270-1280.

52. Eberlein U, Cremonesi M, Lassmann M. Individualized dosimetry for theranostics: necessary, nice to have, or counterproductive? J Nucl Med. 2017;58(suppl 2):97S-103S.

53. Bodei L, Cremonesi M, Ferrari M, et al. Long-term evaluation of renal toxicity after peptide receptor radionuclide therapy with ${ }^{90} \mathrm{Y}$-DOTATOC and ${ }^{177} \mathrm{Lu}-$ DOTATATE: the role of associated risk factors. Eur J Nucl Med Mol Imaging. 2008;35:1847-1856.

54. Vyakaranam AR, Crona J, Norlen O, et al. Favorable outcome in patients with pheochromocytoma and paraganglioma treated with ${ }^{177}$ Lu-DOTATATE. Cancers (Basel). 2019;11:909.

55. Nakazawa A, Higuchi T, Oriuchi N, Arisaka Y, Endo K. Clinical significance of 2F-18 fluoro-2-deoxy-D-glucose positron emission tomography for the assessment of I-131-metaiodobenzylguanidine therapy in malignant phaeochromocytoma. Eur J Nucl Med Mol Imaging. 2011;38:1869-1875.

56. Brabander T, van der Zwan WA, Teunissen JJM, et al. Long-term efficacy, survival, and safety of $\left[{ }^{177} \mathrm{Lu}-\mathrm{DOTA}{ }^{0}, \mathrm{Tyr}^{3}\right]$ octreotate in patients with gastroenteropancreatic and bronchial neuroendocrine tumors. Clin Cancer Res. 2017;23:4617-4624.

57. Strosberg J, El-Haddad G, Wolin E, et al. Phase 3 trial of ${ }^{177}$ Lu-Dotatate for midgut neuroendocrine tumors. N Engl J Med. 2017;376:125-135.

58. Valkema R, Pauwels SA, Kvols LK, et al. Long-term follow-up of renal function

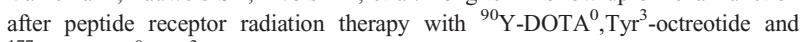
${ }^{177}$ Lu-DOTA ${ }^{0}$, Tyr $^{3}$-octreotate. J Nucl Med. 2005;46(suppl 1):83S-91S.

59. Nastos K, Cheung VTF, Toumpanakis C, et al. Peptide receptor radionuclide treatment and ${ }^{131} \mathrm{I}-\mathrm{MIBG}$ in the management of patients with metastatic/progressive phaeochromocytomas and paragangliomas. J Surg Oncol. 2017;115:425-434.

60. Kong G, Grozinsky-Glasberg S, Hofman MS, et al. Efficacy of peptide receptor radionuclide therapy for functional metastatic paraganglioma and pheochromocytoma. J Clin Endocrinol Metab. 2017;102:3278-3287.

61. Bodei L, Kidd M, Paganelli G, et al. Long-term tolerability of PRRT in 807 patients with neuroendocrine tumours: the value and limitations of clinical factors. Eur J Nucl Med Mol Imaging. 2015;42:5-19.

62. van Essen M, Krenning EP, Kooij PP, et al. Effects of therapy with ${ }^{177}$ Lu-DOTA $^{0}$,$\mathrm{Tyr}^{3}$ octreotate in patients with paraganglioma, meningioma, small cell lung carcinoma, and melanoma. J Nucl Med. 2006;47:1599-1606.

63. Imhof A, Brunner P, Marincek N, et al. Response, survival, and long-term toxicity after therapy with the radiolabeled somatostatin analogue $\left[{ }^{90} \mathrm{Y}\right.$-DOTA $]$-TOC in metastasized neuroendocrine cancers. J Clin Oncol. 2011;29:2416-2423.

64. Lindenberg L, Carrasquillo J, del Rivero J, et al. Comparison of ${ }^{18} \mathrm{~F}-\mathrm{FDG}$ and ${ }^{68} \mathrm{Ga}$-DOTATATE before and after two cycles of PRRT [abstract]. $\mathrm{J}$ Nucl Med. 2019;60(suppl 1):1521.

65. Jha A, Taieb D, Carrasquillo JA, et al. High-specific-activity- ${ }^{131}$ I-MIBG versus ${ }^{177}$ Lu-DOTATATE targeted radionuclide therapy for metastatic pheochromocytoma and paraganglioma. Clin Cancer Res. 2021;27:2989-2995.

66. Carrasquillo JA, Pandit-Taskar N, Chen CC. I-131 metaiodobenzylguanidine therapy of pheochromocytoma and paraganglioma. Semin Nucl Med. 2016;46:203-214. 